\title{
GENETIC DIVERSITY AMONG ASPARAGUS SPECIES AND CULTIVARS USING SSR MARKERS
}

\author{
Idrees, M., M. Irshad, M. L. Pathak ${ }^{1}$, A. Tariq and R. Naeem \\ Department of Biotechnology and Genetic Engineering, Kohat University of Science and Technology \\ (KUST). Kohat, Pakistan; CAS Key Laboratory of Mountain Ecological Restoration and Bioresource \\ Utilization and Ecological Restoration and Biodiversity Conservation Key Laboratory of Sichuan \\ Province, Chengdu Institute of Biology, Chinese Academy of Science, P.O Box 416, Chengdu 61004, \\ China; ${ }^{1}$ The University of Chinese Academy of Science, Beijing, China
}

\begin{abstract}
The aim of the present study was used to develop SSR markers and to determine genetic relationships among Asparagus species and its cultivars. We mined 72953 Asparagus nucleotide sequences from NCBI and were analyzed. In total, 143 SSRs from 1943 SSRs containing sequences were identified. Of these, $13.6 \%$ were dinucleotide repeats and $2.3 \%$ were trinucleotide repeats. The most frequent dinucleotide repeat motif was AA/TT $(73.9 \%)$. The percentage of tri-nucleotide motifs was highest which coded for stop codon (36\%), whereas the Glycine was least present (4.5\%). Among the total of 14 SSR primers used, 10 markers yielded 144 (92.3\%) polymorphic bands with an average of 14.4 alleles per primer. Cluster analysis based on UPGMA grouped the Asparagus species and its cultivars into two main clusters. Cluster A contained only A. grecilius which is more diverse than others, while cluster B was further clustered into two sub clusters. Cluster I was comprised of Asparagus officinalis cultivars and cluster II was comprised of Asparagus wild species. Furthermore, these primers $(42.8 \%)$ were found to be transferable in other medicinal plants (Curcuma longa L. and Saussurea costus). The results suggest that SSR markers are sufficiently useful and powerful to assess genetic relationships and diversity analysis in Asparagus and A. officinalis cultivars. Furthermore, these markers will be particularly useful for evolutionary and genetic mapping studies in Asparagus.
\end{abstract}

Keywords: Asparagus, Simple Sequence Repeats, genetic diversity, Saussurea costus, Curcuma longa.

\section{INTRODUCTION}

Asparagus Lindley is a genus of dioecious plants with approximately 150 species throughout the world. The genus has been moved from the family Liliaceace to a newly created family Asparagaceae (Kubitzki and Rudall 1998). It is grown in a wide range of environments including temperate regions, humid tropics and arid tropics up to an altitude of $1200 \mathrm{~m}$ (Dutta 2007). Asparagus remains valuable as plant species from eras having both therapeutic and nutraceutical importance as well as for food consumption (Shasnay et al. 2003). Asparagus contains fructans and saponin that have antitumor activity and reduce the risk of disorder such as diarrhea, constipation and helps in diseases like osteoporosis, obesity, cardiovascular disease, rheumatism and diabetes. The root of Asparagus possesses antispasmodic, aphrodisiac, diuretic, demulcent and galactagogue properties and has been commonly used for different medicinal purposes. Its roots are also useful in diseases of liver and kidney, gonorrhea, epilepsy, jaundice and disease of blood and eyes. Internally it is used in the treatment of infertility, threatened miscarriage, menopausal problems, stomach ulcer, hyperacidity and loss of libido, while externally it is used to treat stiffness in joints. It also stimulates insulin action, secretion and inhibits starch digestion (Mandal et al. 2000).

Simple sequence repeats (SSRs) are stretches of genomic DNA that consist of short tandem repeated motifs of 1-6 nucleotides, and can be found in high frequency of most taxa nuclear genomes (Beckmann and Weber 1992). SSRs are more desirable because of their reproducibility, codominant inheritance, multiallelic nature, high abundant and well genetic coverage, which makes the method highly polymorphic and specific (Bornet and Branchard 2001). These are a PCR-based technique amenable to 
automation and require short period of time for their analysis. SSRs are widely used in various aspect of molecular genetics including fingerprinting, marker assisted selection, genetic map development, gene flow characterization, linkage analysis and genetic diversity (Koppolu et al. 2010). The conserved flanking sequence of specific SSR loci in genome are shown to be conserved within taxa, genus and even among closely related genera (Varshney et al. 2002). SSR sequences frequently change by proofreading errors and slippage during DNA replication and thus changed the length of the repeats (Eisen 1999). However, genomic SSR markers development is expensive and time consuming, particularly if developed from genomic libraries. These SSRs markers previously used to analyze plant genetic diversity (Hafezi-Shahroodian et al. 2011). During the past few years, In-silico SSR-mining has gone through a rapid evolution. With much advancement in bioinformatics and genomics, various insilico approaches are being used increasingly. These tools were used to design for the development of SSR markers in a very short span of time. These bioinformatics approaches can eliminate the need for screening and costly library construction. Mining SSR from NCBI databases has been streamlined with technological advance to make the process more efficient, cheaper and successful and proved to be an effective approach to develop microsatellites markers for genetic mapping and genetic diversity (Portis et al. 2007). In summary, varietal characterization and taxonomy are difficult in Asparagus, so molecular markers have proven useful. To the best of our knowledge, there is no study using SSRs markers to evaluat genetic diversity among Asparagus species and its cultivars in Pakistan. The aim of the current study was to examine (1) frequency and distribution of SSRs (2) Nature and distribution of encoded amino acid (3) BLAST analysis of amplified markers and 4) to develop SSRs primer and validate their polymorphism/ transferability in other medicinal plants.

\section{MATERIAL AND METHODS}

All nucleotide sequences of Asparagus were retrieved in Fasta format from National Center Biotechnology Information (NCBI) database. The nucleotide sequences of Asparagus were aligned using Clustal X and screened for the presence of Simple Sequence Repeats using microsatellite search module "MISA" which include di, tri and tetra- nucleotide repeats in the Search. These were assembled using CAP3 programme (Huang and Madan 1999).

Table 1. Plant collections and specimen of Asparagus species.

\begin{tabular}{|c|c|c|c|c|}
\hline Species and Cultivars & Collection sites & Altitude (m) & Longitude (E) & Latitude $(\mathbf{N})$ \\
\hline A. racemosus wild & Charbhage, Swat & 909 & $72^{\circ} 21^{\prime} \mathrm{E}$ & $34^{\circ} 46^{\prime} \mathrm{N}$ \\
\hline A. capitatus wild & Ghalegay, Swat & 950 & $72^{\circ} 12^{\prime}$ and $72^{\circ} 32^{\prime} \mathrm{E}$ & $35^{\circ} 20^{\prime}$ to $35^{\circ} 48^{\prime} \mathrm{N}$ \\
\hline A. gracelus wild & Shamozu, Swat & 465 & $72^{\circ} 12^{\prime} \mathrm{E}$ & $34^{\circ} 68^{\prime} \mathrm{N}$ \\
\hline A. adscenden wild & Jerma, Kohat & 508 & $71^{\circ} 29^{\prime} \mathrm{E}$ & $30^{\circ} 40^{\prime} \mathrm{N}$ \\
\hline A. setaceus & Bhage Jinnah, Lahore & 209 & $74^{\circ} 33^{\prime} \mathrm{E}$ & $31^{\circ} 54^{\prime} \mathrm{N}$ \\
\hline A. densiflorus & More green, Lahore & 217 & $74^{\circ} 34^{\prime} \mathrm{E}$ & $31^{\circ} 54^{\prime} \mathrm{N}$ \\
\hline A. plumosus & Mingora, Swat & 950 & $72^{\circ} 26^{\prime} \mathrm{E}$ & $34^{\circ} 69^{\prime} \mathrm{N}$ \\
\hline A. officinalus & NARC, Islambad & 508 & $73^{\circ} 10^{\prime} \mathrm{E}$ & $33^{\circ} 42^{\prime} \mathrm{N}$ \\
\hline A. officinalis $C v$ Abril & ARI, Mingora & 984 & $72^{\circ} 26^{\prime}$ and $72^{\circ} 36^{\prime} \mathrm{E}$ & $34^{\circ} 68^{\prime} \mathrm{N}$ \\
\hline A. officinalis Cv Apollo & ARI, Mingora & 984 & $72^{\circ} 26^{\prime}$ and $72^{\circ} 36^{\prime} \mathrm{E}$ & $34^{\circ} 68^{\prime} \mathrm{N}$ \\
\hline A. officinalis $C v$ Gersengum & ARI, Mingora & 984 & $72^{\circ} 26^{\prime}$ and $72^{\circ} 36^{\prime} \mathrm{E}$ & $34^{\circ} 68^{\prime} \mathrm{N}$ \\
\hline A. officinalis $C v$ Huchel & ARI, Mingora & 984 & $72^{\circ} 26^{\prime}$ and $72^{\circ} 36^{\prime} \mathrm{E}$ & $34^{\circ} 68^{\prime} \mathrm{N}$ \\
\hline A. officinalis $C v$ Taranga & ARI, Mingora & 984 & $72^{\circ} 26^{\prime}$ and $72^{\circ} 36^{\prime} \mathrm{E}$ & $34^{\circ} 68^{\prime} \mathrm{N}$ \\
\hline A. officinalis $C v$ Para selection & ARI, Mingora & 984 & $72^{\circ} 26^{\prime}$ and $72^{\circ} 36^{\prime} \mathrm{E}$ & $34^{\circ} 68^{\prime} \mathrm{N}$ \\
\hline Saussurea costus & Shangla & 1904 & $72^{\circ} 35^{\prime} \mathrm{E}$ & $34^{\circ} 53^{\prime} \mathrm{N}$ \\
\hline Curcuma longa L. & Bannu & 371 & $70^{\circ} 18^{\prime} \mathrm{E}$ & $33^{\circ} 00^{\prime} \mathrm{N}$ \\
\hline
\end{tabular}

The SSRs primers were designed to the nucleotide sequences of Asparagus having SSRs motifs using primer3 software, Primer design criteria included, minimum of five or six di, tri, and tetra- 
nucleotide repeats. Simple Sequence Repeats were masked as target region including amplicon size ranges from $200-300 \mathrm{bp}, 55-60^{\circ} \mathrm{C}$ of optimal annealing temperature, average GC content ranges from 50 to $60 \%$, and length of primer ranges from 18 to $24 \mathrm{bp}$. The output was displayed as sequence of forward and reverse primers. The Primers were synthesized by Invitrogen Corp. CA, USA. SSR loci putative functions were allowed by similarities with the non-redundant sequence database using BLASTX software (Altschul et al. 1997).

A total of 14 different Asparagus species and A. officinalis cultivars was collected from different regions of Pakistan including Islamabad, Lahore, Kohat and Swat (Table 1 and Fig. 1A). Leaves were collected and immediately stored in silica bags.

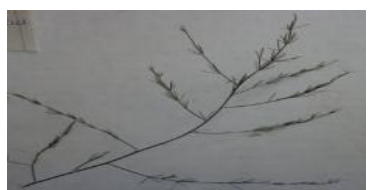

a

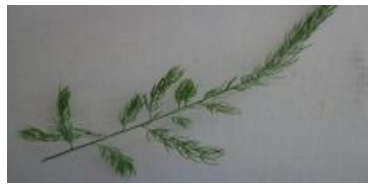

e

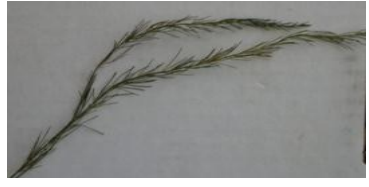

i

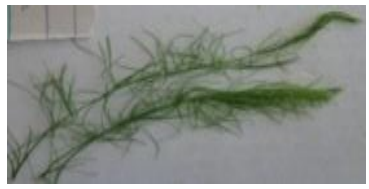

m

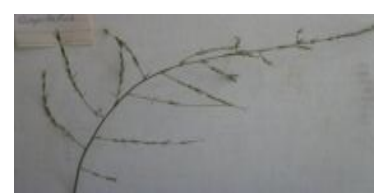

b

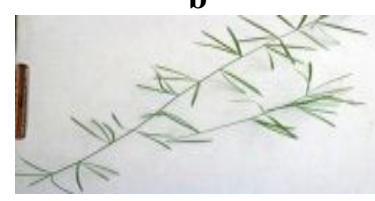

f

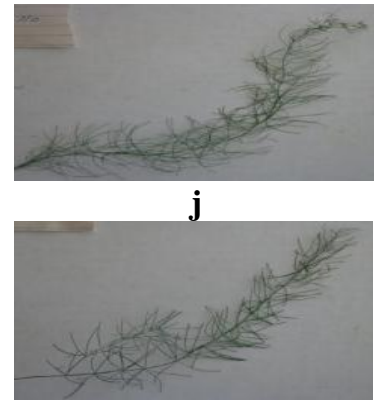

n

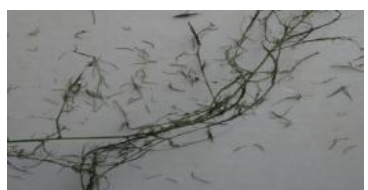

c

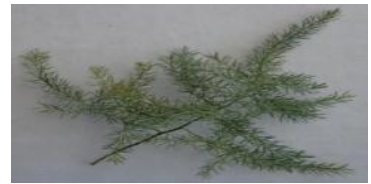

g

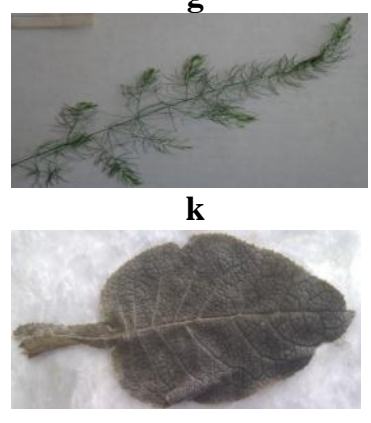

o

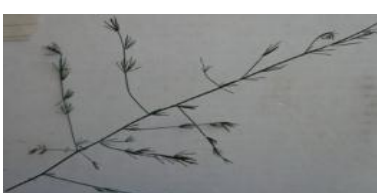

d

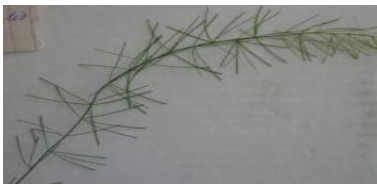

h

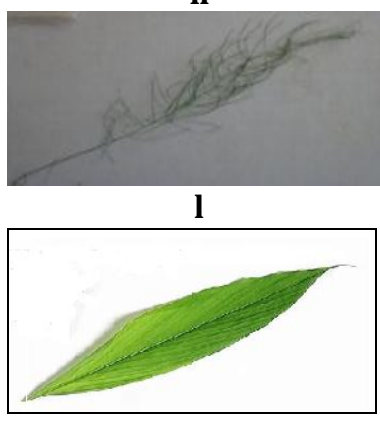

p

Fig. 1A. Specimen of Asparagus species: a. A. racemosus; b. A .capitatus; c. A. gracelus; d. A. adscenden; e. A.setaceus; f. A.densiflorus; g. A.plumosus; h. A. officinalus; i. A. officinalis; j. A. officinalis; k. A. officinalis; 1. A. officinalis; m. A. officinalis; n. A. officinalis; o. Saussurea costus; and p. Curcuma longa.

Genomic DNA was extracted from the fresh and young leaves of Asparagus using standard CTAB method (Cetyl trimethyl ammonium bromide) as described by Doyle and Doyle (1987) with slight modifications, i.e the addition of high concentration of PVP (polyvinyl-pyrrolidine). Fresh leaves of Asparagus $(100 \mathrm{mg})$ were ground well using pre-warmed solution containing $800 \mu \mathrm{l}$ extraction buffers. After grinding, $20 \mu \mathrm{l}$ of $\beta$-mercaptoethanol was added and shaken vigorously by inversion to produce slurry. The tube was then incubated at $65^{\circ} \mathrm{C}$ for 30 minutes with intermittent shaking and vortexing every 5 minutes. The tube was kept at room temperature $\left(25^{\circ} \mathrm{C}\right)$ for 7 minutes and then $800 \mu l$ of chloroform: isoamylalchol (24:1) was added.

The solution was mixed by inversion for 10 - 15 minutes and kept at room temperature for 7 minutes. The emulsion was centrifuged at $12,000 \mathrm{~g}$ at $24^{\circ} \mathrm{C}$ for 10 minutes and the upper layer was transferred to another sterile tube. The chloroform: isoamylalchol step was repeated again and the supernatant was centrifuged at 12,000 g for 10 minutes. The upper aqueous phase was placed in a sterile tube. The DNA was precipitated by adding equal volume $(0.6 \mathrm{~V})$ of chilled isopropanol and $30 \mu \mathrm{l}$ of $5 \mathrm{M}$ $\mathrm{NaCl}$ and then was kept at $-20^{\circ} \mathrm{C}$ for one hour. The sample was centrifuged at $12,000 \mathrm{~g}$ for 10 minutes at $4^{\circ} \mathrm{C}$. The supernatant was discarded and the DNA pellet was first washed with $800 \mu \mathrm{l}$ of wash buffer 
(Tris $\mathrm{HCl} 5 \mathrm{mM}$, Nacl $25 \mathrm{mM}$ and Etnaol 25\%) and then with $300 \mu \mathrm{l}$ of $70 \%$ ethanol. The pellet was air dried at room temperature for 15 minutes and finally the purified DNA pellet was allowed to dissolved in $30 \mu \mathrm{l}$ of TE buffer and stored at $-20^{\circ} \mathrm{C}$.

For polymorphism and transferability study, PCR amplifications were carried out in Veriti 96 well thermal cycler (Applied Biosystem Inc, USA) using SSRs markers. Amplifications were carried out in $25 \mu \mathrm{l}$ master mix containing $2.5 \mu \mathrm{l}$ of $1 \mathrm{X}$ PCR buffer; $0.2 \mathrm{mM}$ dNTPs mix; $2.5 \mathrm{mM} \mathrm{MgCl} 2 ; 1$ Unit of Taq DNA polymerase (Invitrogen); $0.2 \mu \mathrm{M}$ of each forward and reverse primers and $2 \mu \mathrm{l}$ of $50 \mathrm{ng}$ of template DNA. Conditions for amplification of the region consists of initial denaturation at $95^{\circ} \mathrm{C}$ for 5 minutes and then 35 cycles of denaturation at $94^{\circ} \mathrm{C}$ for 1 minute (denaturation), annealing of primer at $55^{\circ} \mathrm{C}$ for 1.5 minutes, $72^{\circ} \mathrm{C}$ for 1.5 minutes (extension), with the final step of extension at $72^{\circ} \mathrm{C}$ for 7 minutes followed by hold temperature at $4^{\circ} \mathrm{C}$. The PCR products were separated on $2 \%$ Agarose gel in Tris-Borate EDTA buffer and stained with $0.5 \mathrm{mg} / \mathrm{ml}$ ethidium bromide. These were visualized and photographed under a UV transilluminator (WiseDoc). The sizes of the DNA products were calculated by comparison with $1 \mathrm{~kb}$ DNA ladder.

Analysis of the electrophoresis patterns of each SSR primers were scored manually as presence or absence of bands. The results were analyzed on the principle that a band is considered to be 'polymorphic' if it is present in some individuals and absent in others, and 'monomorphic' if present in all individuals. Numerical Taxonomy System (NT-SYS), version 2.11 from applied biostatis Inc. (2002), was used to analyze the results obtained from scoring. For measuring genetic similarity and distance among Asparagus species and cultivars, Nei and Li genetic similarity coefficient was used (Nei and Li 1979). Cluster analysis was performed by UPGMA (Unweighted Pair wise Methods with Arithmetic averages) to generate a dendogram.

\section{RESULTS AND DISCUSSION}

Microsatellite markers were considered as the best genetic markers for the analysis of genetic variation and genetic improvement of plants in comparison to other available markers. Conventional methods for the development of SSR markers were costly and time consuming. However the aim of the present study was to develop cost efficient method by retrieving nucleotide sequences from NCBI database that containing microsatellite to produce SSR markers in Asparagus and to evaluate the genetic relationship among Asparagus species and its cultivars in Pakistan using SSR markers. Several studies have used traditional approaches for microsatillete markers evolution in various plants species (Thiel et al. 2003). Previously, EST-SSR markers have been developed in Asparagus (Caruso et al. 2008).

Table 2. Summary of SSRs in Asparagus.

\begin{tabular}{lc}
\hline Screening of Parameter & Generated data by SSR Finder \\
\hline Total examined Sequences & 72953 \\
Nucleotide sequence size & 204609 \\
SSR identified & 1915 \\
Sequence containing SSR & 143 \\
SSR containing more than 1 sequence & 96 \\
Dinucleotide & 261 \\
Trinucleotide & 44 \\
Tetranucleotide & 16 \\
Pentanucleotide & 5 \\
\hline
\end{tabular}

SSR markers were developed from 72953 nucleotide sequences on NCBI by In-silico methods. The frequency of SSRs (91.4\%) recognized in our study is much higher as compared to previous studies in Catharanthus roseus (10.2\%). The difference in the frequencies of SSRs detected depends on the search 
module used for SSRs type motif, sequence size analysis and tool for mining database (Portis et al. 2007, Gupta and Prasad 2009).

From 261(13.6\%) SSR containing sequences, di-nucleotide was the most abundant which account for 13.6 percent, followed by tri-nucleotide, tetra-nucleotide and penta-nucleotide which were 2.3, 0.84 and $0.27 \%$, respectively (Table 2), similar observations were reported in the plant of Nelumbo nucifera (Pan et al. 2010). In few studies of animals, dinucleotide repeat was the dominant type reported. The most frequent dinucleotide repeat motifs were AA/TT (73.9\%), followed by AT/AT (17.6\%), and AG/CT (2.68\%). Similar results have been reported in other taxa of plant genomes (Kumpatla and Mukhopadhyay 2005). The dinucleotide motif TA/TA (1.18\%) was found as least prevalent while the AT motif was found to be more frequent in plant genome, similarly AG/CT motif was represented as codons GAG, UCU, and CUC in mRNA populations which were further translated into the amino acids Argnine, Alanine, and Leucine, respectively. Alanine and Leucine occurred at high frequency of 8 to10 $\%$ in all proteins (Wen 2010). Among the tri-nucleotide repeats, motif AAA/TTT (50\%) was most common, followed by ATT/GCG (36.3\%) and CCT/TCC (9\%). The most common tetra-nucleotide motif was AAAA/TTTT (62.5\%) followed by GAGA/CTCT (37.5\%) and ATAT/ATAT (6.25\%) (Fig. $1 \mathrm{a}, \mathrm{b}, \mathrm{c}, \mathrm{d})$.

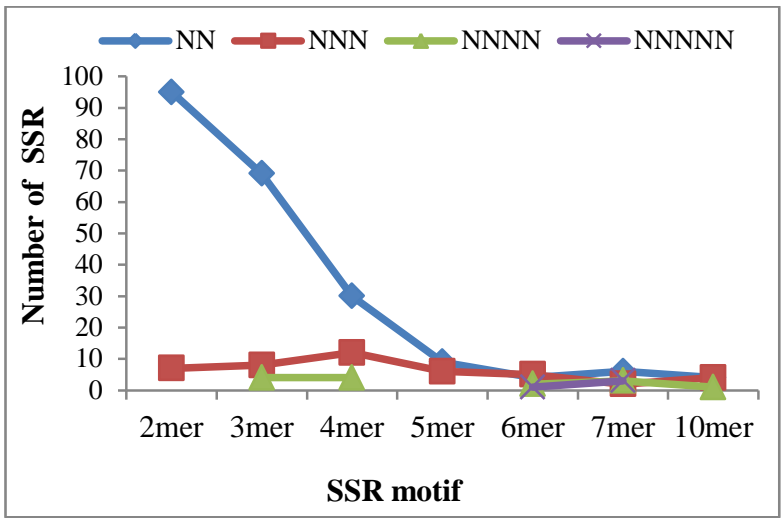

$\mathbf{a}$

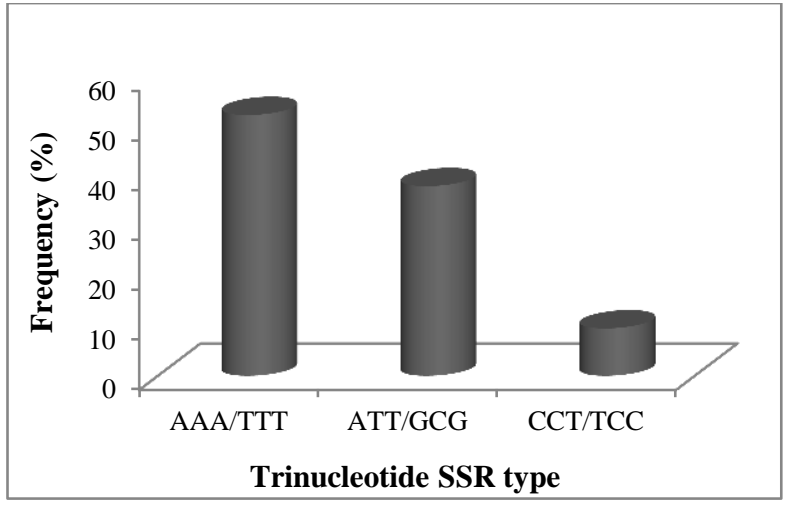

c

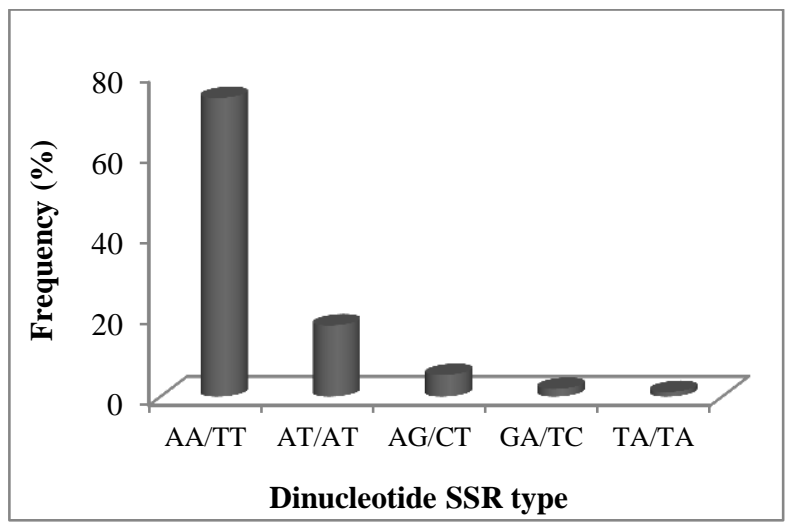

b

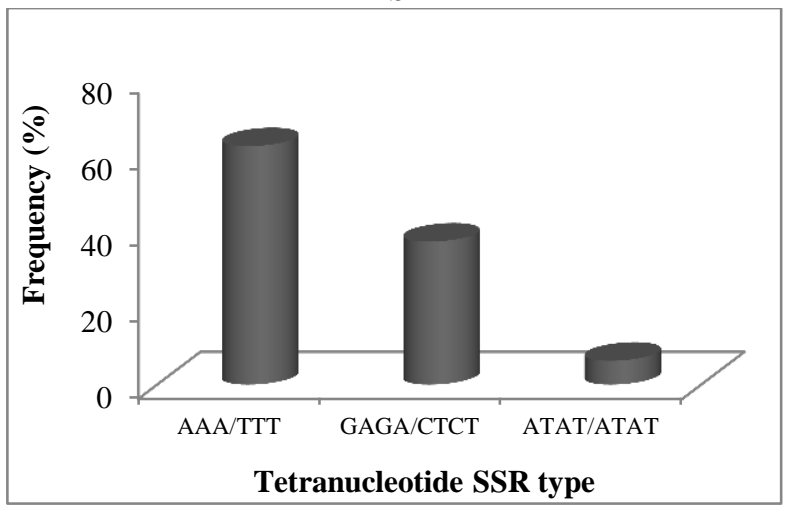

d

Fig. 1. Frequency distribution of identified SSR sequence: a. all different nucleotide repeats in Asparagus; b. dinucleotide; c. Trinucleotide; and d. Tetranucleotide.

Each of the tri-nucleotide motifs in SSR loci is coded for a specific amino acid which possesses several biological functions. In the present study, 44 tri-nucleotide repeats motif was found, which code for 4 amino acids, viz. lysine, phenylalanine, arginine and glysine, and one stop codon. The percentage of tri-nucleotide motifs was highest which coded for stop codon (36 \%), followed by Lysine (31 \%), Phenylalanine (21.4\%), and Arginine (7.1\%), while the Glycine was least present (4.5\%) (Fig. 2). 


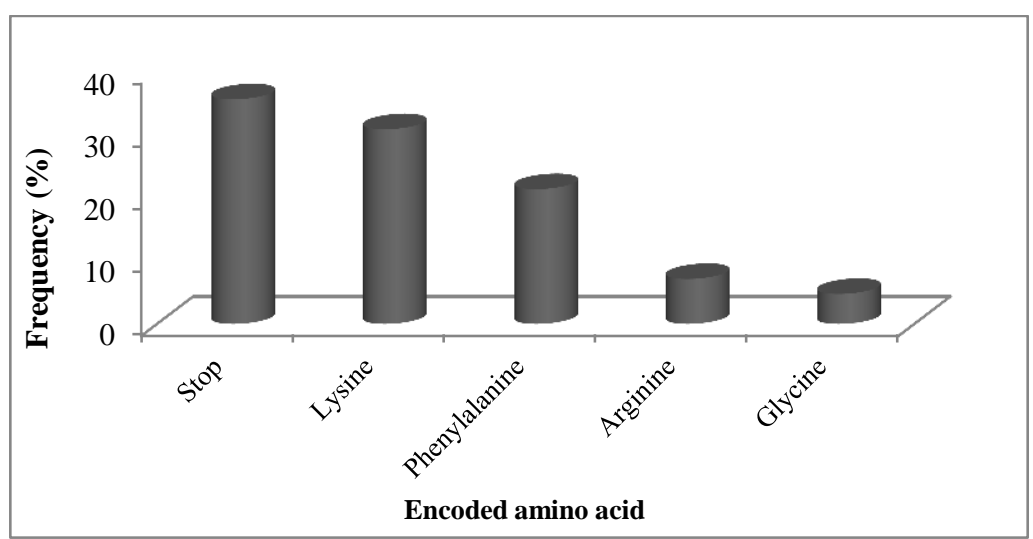

Fig. 2. Distribution of SSR encoded amino acid in Asparagus

Arginine in the present result was $21.4 \%$ that similar to the previously studied in Arabidopsis, Gnetum, Oryza and Pinus, which showed that the predominant amino acid was Arginine (Victoria et al. 2011). Till date, the amino acid distribution in SSR loci in Asparagus has not reported yet. These encoded amino acids of SSR were divided further into 4 categories, (a) hydrophobic and hydrophilic amino acid, (b) polar and non polar amino acid, (c) acidic, basic and neutral amino acid, and (d) aliphatic and aromatic amino acid (Fig. 3a, b, c, d).

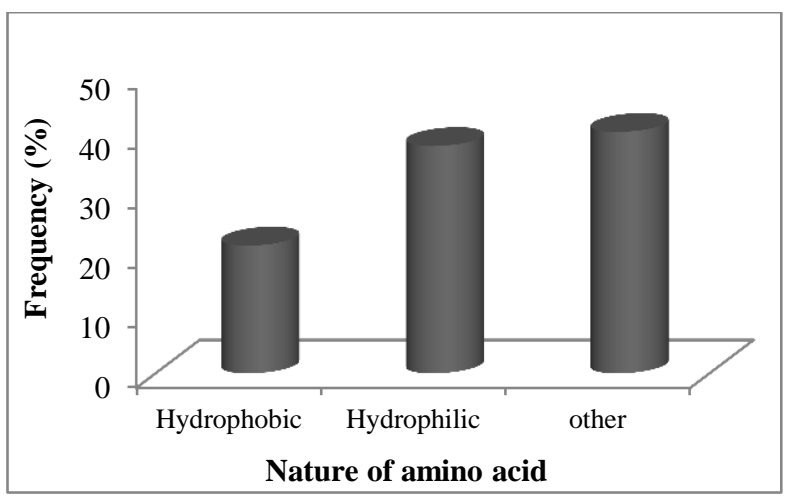

a

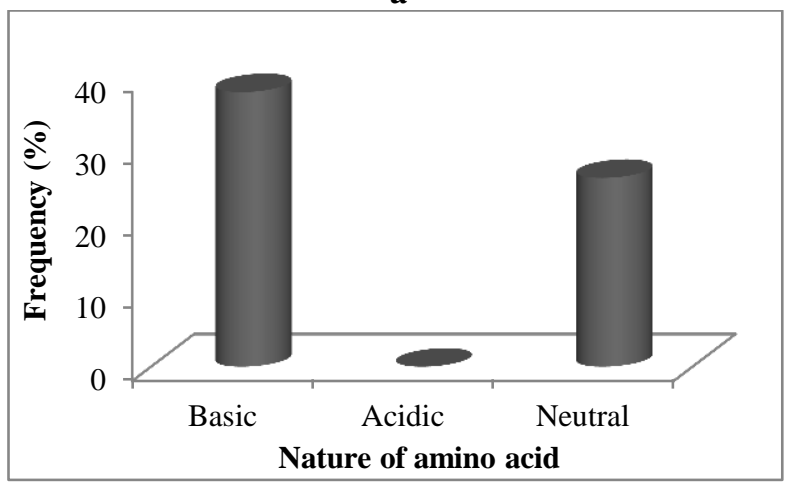

c

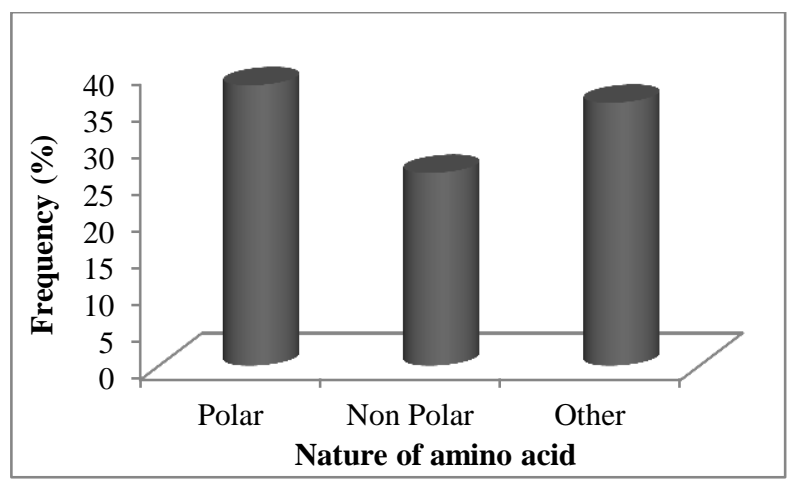

b

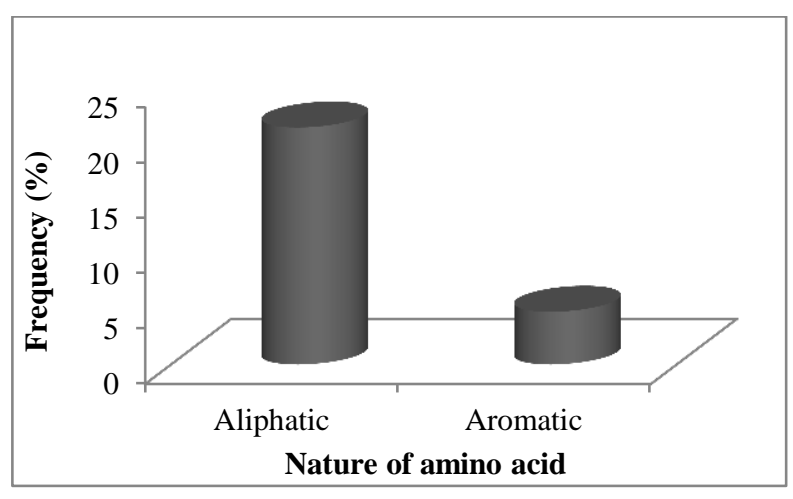

d

Fig. 3. Distribution of SSR encoded amino acid in Asparagus: a. hydrophobic and hydrophilic amino acid; b. polar and non polar amino acid; c. acidic, basic and neutral amino acid; and $\mathbf{d}$. aliphatic and aromatic. 
From 143 sequences containing SSR, 40 (28.0 \%) nucleotide sequences were allowed for primer designing, while remaining 103 (72\%) sequences were unsuitable for primer construction because they have SSR motifs either at the beginning or at the end of nucleotide sequence. Out of 40, 25 primer pairs were developed successfully by utlizing primer 3 softwares (Rozen and Skaletsky 2000). Finally, subsets of 14 primer pairs were selected on the basis of product size for the justification of amplification and evaluation of genetic variations in Asparagus species and its cultivars (Table S1).

Table S1. Characterization of 14 SSRs developed from Asparagus.

\begin{tabular}{|c|c|c|c|c|c|c|c|}
\hline Primers & ID & SSR motifs & $\begin{array}{c}\text { Tmp. } \\
\left({ }^{\circ} \mathrm{C}\right)\end{array}$ & GC\% & Forward primer & Reverse primer & $\begin{array}{l}\text { Product } \\
\text { size (bp) }\end{array}$ \\
\hline ndhf-rpl32 & gi|327442444 & $(\mathrm{AT})_{4},(\mathrm{AA})_{8}$ & 58.98 & 39.05 & TTCAAATTTTCCСCTCCTTTC & TTGCTCAAAATGGGTCATTC & 235 \\
\hline rps16-trnK & gi| 327442522 & $(\mathrm{AA})_{4}$ & 60.3 & 44.05 & TTCCTTGAAAAAGGTGCTCAA & GGTGGATTCCCACAACAAGA & 207 \\
\hline trnQ-rps16 & gi|327442389 & $(\mathrm{TT})_{4}$ & 60.3 & 38.75 & ATGATTCACCATCCCGAAAA & TGAATAGTCATTGGATCAACGGTA & 197 \\
\hline psbD-trnT & gi|327442494 & $(\mathrm{AA})_{5}$ & 59.8 & 42.5 & CGTCCAATGCCCTTTACAAT & AATTTAGGGGCAGGGAAAAA & 227 \\
\hline atpB-rbcl & gi|37721940 & $(\mathrm{AT})_{4}$ & 59.83 & 43.93 & TCTCATTGGCTGCTGTTCTTT & ATATATGGCGCAACCCAATC & 245 \\
\hline petB & gi|56603578 & $(\mathrm{TT})_{4}$ & 60 & 43.75 & AAGAGGCCTGTAACGAGCAA & CACAAATACTGATTTCACCGGATA & 183 \\
\hline matK & gi|374975311 & $(\mathrm{TT})_{4}$ & 60.2 & 47.5 & CCAAAATCGATTCGTTGGAC & CATATGGATGGGATGGGGTA & 247 \\
\hline A synth & gi|16075 & $(\text { TTGGT })_{4}$ & 60 & 45 & GCTGGTCGAACAATTTGGAT & ATACAACGCCCCACACATTT & 235 \\
\hline rpl32-trnL & gi|327442338 & $(\mathrm{AT})_{5},(\mathrm{AA})_{4}$ & 59.7 & 35.2 & TCTCTTTGACGATTTGTTTTTCA & CCGGAACATTTGGAATTTGA & 154 \\
\hline ZHD1 & gi||164562228 & $(\mathrm{TT})_{10}$ & 60 & 47.5 & GGAAGAGGGTGCGTGTTTTA & AAACGAACCAAAGTGCCATC & 201 \\
\hline ITS $1 \& 2$ & gi| 319432428 & $(\mathrm{CC})_{9 *}$ & 60 & 50 & CCGTGAACCATCGAGTCTTT & CAGCGTCTTTTGTCTGTCCA & 239 \\
\hline AoAS1 & gi|383212088 & $(\mathrm{TC})_{4}$ & 60 & 52.5 & CTCATGCCCACTCCGATATT & TCAGCCTCCACGAACTCTCT & 220 \\
\hline AG7 & gi|52846592 & $(\mathrm{AG})_{10}$ & 59.6 & 47.5 & TTTTGCTCCGATCATTTTCA & CCTCTTCGTCTTCATCAGCC & 194 \\
\hline TC7 & gi|52845609 & $(\mathrm{TC})_{15}$ & 59.2 & 44 & CGCCCCGAATCAACTAATAA & TACTGCGGAGGTATGTGGGT & 220 \\
\hline
\end{tabular}

Most of the SSR markers developed in Asparagus were not allowed to any kind of function. All amplified SSR marker sequences were compared with non-redundant protein data base using BLASTX 2.2.27 program. Of the 14 primers, seven (50\%) of the developed SSR markers were found to show significant similarities with known protein sequence, which were in close agreement with previous studies, which showed that $46 \%$ of L. Vannamei ESTs sequences corresponded to known proteins (Perez et al. 2005). One of the positive hit was similar to ribosomal protein, two of the positive hits similar to transcription factors, one of the positive hit matched to chloroplast gene, one similar to carbon/nitrogen metabolism while two of the positive hits were matched to hypothetical proteins. The results of the present study revealed that such SSR loci showed sequence similarity with other putative functions of protein sequence, which suggested these SSR loci can be used to saturate various plants metabolic pathways (Victoria et al. 2011). Putative function of SSR markers are illustrated in Table 3.

Table 3. Putative function of seven amplified SSR markers.

\begin{tabular}{|c|c|c|c|c|c|}
\hline Primer & $\begin{array}{l}\text { Gene bank } \\
\text { Accession no. }\end{array}$ & Putative function & Species & E-value & Score \\
\hline ZHD1 & EU200163.1 & Transcription factor, putative & Ricinus communis & $1 \mathrm{e}-68$ & 235 \\
\hline A. synthase & X67958.1 & glutamine-dependent asparagine synthetase & Triticum aestivum & 0 & 1025 \\
\hline rpl32-trnL & AB613848.1 & 50S ribosomal protein $\mathrm{L} 32$ & hoenix dactylifera & $2 \mathrm{e}-08$ & 59.3 \\
\hline matK & HQ180855.1 & maturase K (chloroplast) & Hemiphylacus alatostylus & 0 & 959 \\
\hline AoASI & $\mathrm{AB} 673050.1$ & MYB-related transcription factor PHAN1 & Pisum sativum & $5 e-123$ & 367 \\
\hline rps16-trnK & AB613981.1 & hypothetical protein SORBIDRAFT & Sorghum bicolor & 0.001 & 45.4 \\
\hline AG7 & CV459406.1 & hypothetical protein VITISV_023571 & Vitis vinifera & $1 e-28$ & 116 \\
\hline
\end{tabular}

A total of 14 SSR primers was selected for amplification and polymorphism in Asparagus species and its cultivars (Table 1 and Fig. 1A). In the present investigations, out of total 14 SSR primers used, 10 SSR primers (AA5, TT10, TC15, TTGGT4, AG10, AA4, AT4, TT4, TC4) showed polymorphism 
and produced a total of 156 bands, of which $144(88.36 \%)$ were polymorphic bands with an average of 14.4 alleles per primer (Fig. S1 and Table 4).

Table 4. SSR motifs and polymorphism index obtained for Asparagus species.

\begin{tabular}{lcccc}
\hline Primer & Repeat motifs & No. of bands & Polymorphic bands & Polymorphism Index \\
\hline psbD-trnT & (AA) 5 & 17 & 16 & 94.1 \\
PetB & (TT) 4 & 11 & 9 & 81.8 \\
ZHD1 & (TT) 10 & 11 & 7 & 63.6 \\
TC7 & (TC) 15 & 39 & 39 & 100 \\
A.synthetase & (TTGG) 4 & 16 & 14 & 87.5 \\
AG7 & (AG) 10 & 8 & 7 & 87.5 \\
rps16-trnK & (AA) 4 & 4 & 3 & 75 \\
atpB-rbcL & (AT) 4 & 17 & 16 & 94.1 \\
trnQ-rps16 & (TT) 4 & 17 & 17 & 100 \\
AoAs1 & (TC) 4 & 16 & 16 & 100 \\
\hline Total & & $\mathbf{1 5 6}$ & $\mathbf{1 4 4}$ & $\mathbf{9 2 . 3}$ \\
\hline Average & & $\mathbf{1 5 . 6}$ & $\mathbf{1 4 . 4}$ & $\mathbf{8 8 . 3 6}$ \\
\hline
\end{tabular}

These findings are similar to those of Dow et al. (1995) who observed 14.3 alleles per primer in Oak. Genetic diversity among Asparagus species was also studied by other investigators, Ray et al. (2010) who used 6 ISSR markers for 6 different species of Asparagus. They analyzed a total of 110 ISSR fragments with an average of 27.5 fragments per prime. Ginwal et al. (2011) applied $18 \mathrm{cpSSR}$ markers in which $5(27.77 \%)$ were identified yielding good amplification in A. racemosus. In the present study, the genetic similarity coefficients ranging from 0.52 to 0.94 reveal a high level of genetic variations among Asparagus species which was in close agreement with Pan et al. (2004) who found genetic similarity matrix using RAPD markers which ranged from $60.5 \%$ to $88.5 \%$ for the collection of S. spontaneum clones. The polymorphism observed in present study by using SSR markers for Asparagus species and its cultivars was effective to determine genetic variation at species level (Caruso et al. 2008).

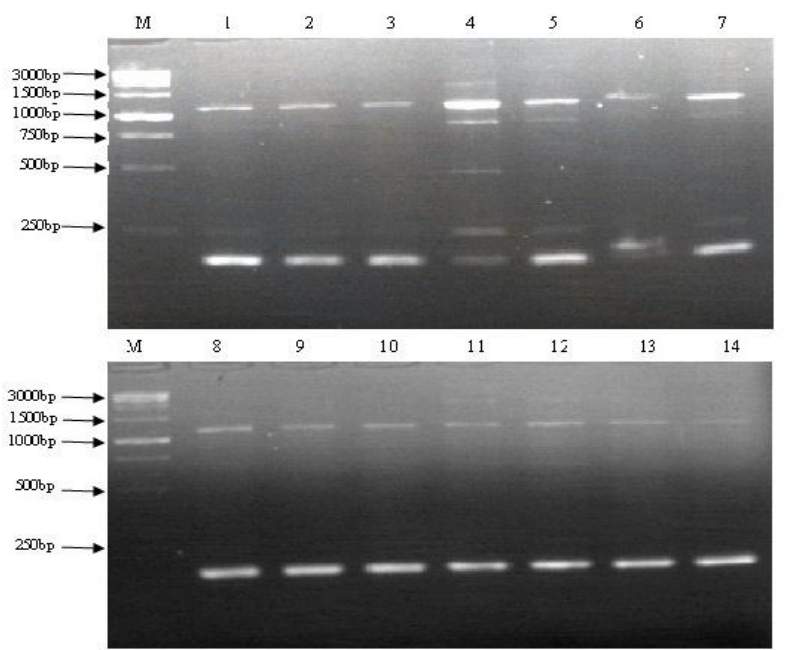

(PetB)

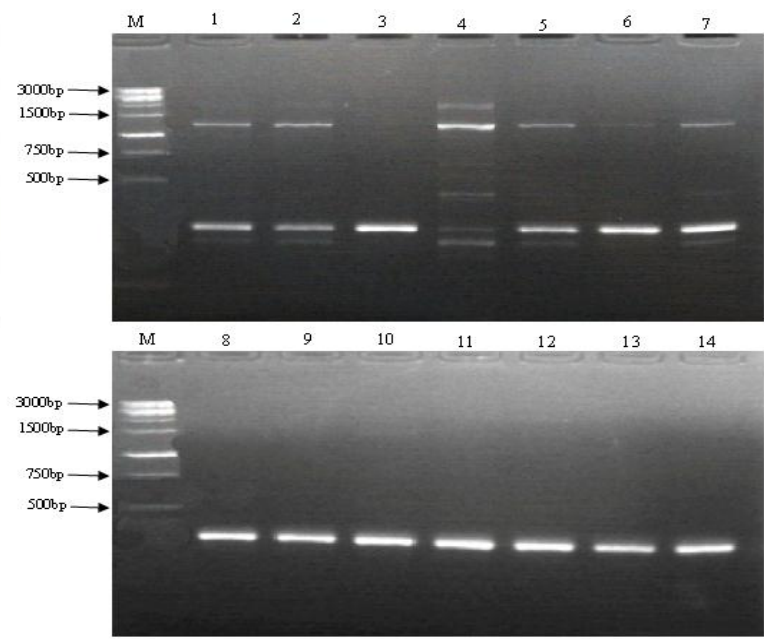

(atpB-rbcL) 


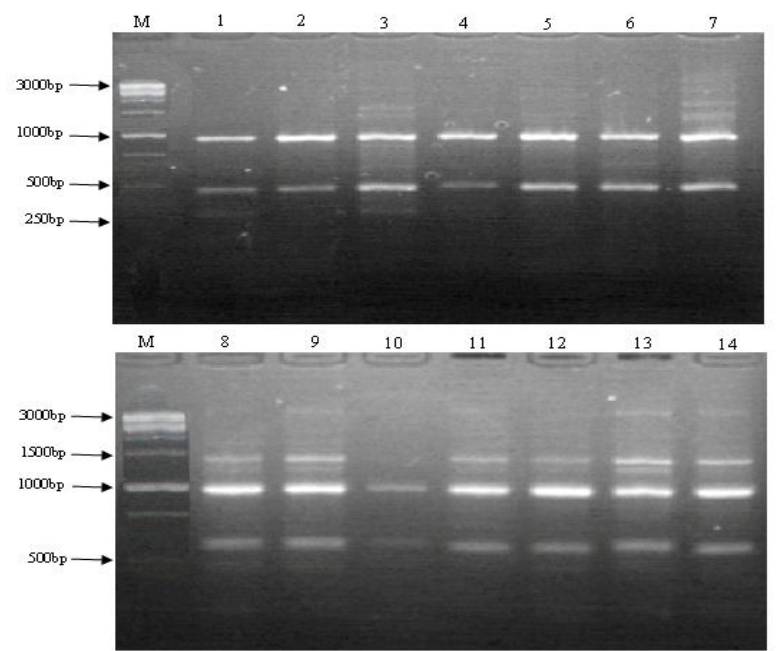

(A. synthetase)

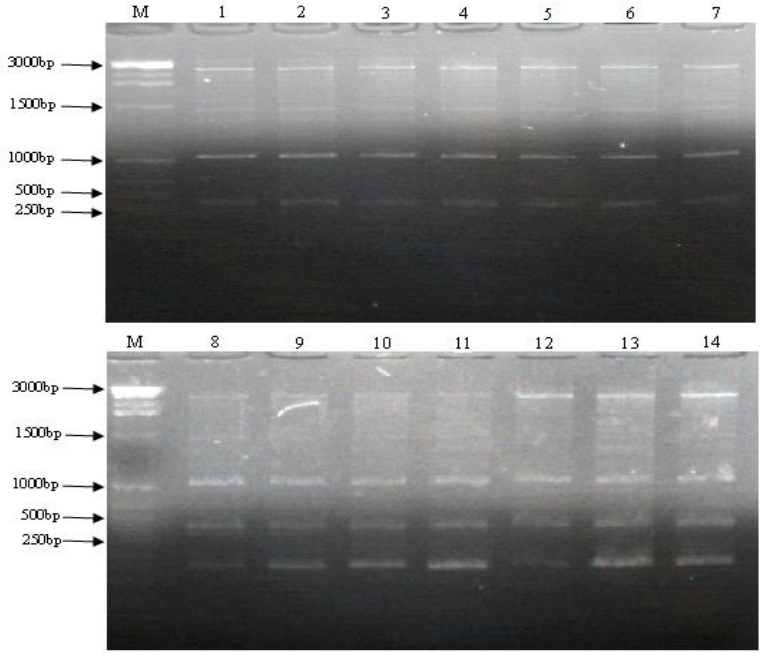

(ZHD1)

Fig. 4. Electrophoretic pattern of SSR product generated with primers (A) A. synthetase, (B) psbD-trnL and (C) petB (D) matK: Lane 1-14 represents 1) A.adscenden, 2) A.racemosus, 3) A.capitatus, 4) A. gracilis, 5) A. densiflorus, 6) A. setaceus, 7) A. plumosus, 8) A.officinalis, 9) A.officinalis cv. Appollo, 10) A.officinalis cv. Abril, 11) A.officinalis cv. Gersengum, 12) A.officinalis cv. Huchels, 13) A.officinalis cv. Para selection 14) A. officinalis cv. Taranga.

Cluster analysis of SSR data using UPGMA revealed Asparagus species and A. officinalis cultivars into 2 main clusters, one cluster comprised of only Asparagus species and other cluster comprized of $A$. officinalis cultivars (Fig. 4). Clustering, using SSR markers, showed that A. gracelis was quite separated from other species genotypes, because of its different genetic background, suggesting that these might be due to mutations, genetic changes and interspecific hybridization. Our result is consistent with Ray et al. (2010) that also cluster Asparagus species into 2 subclusters.

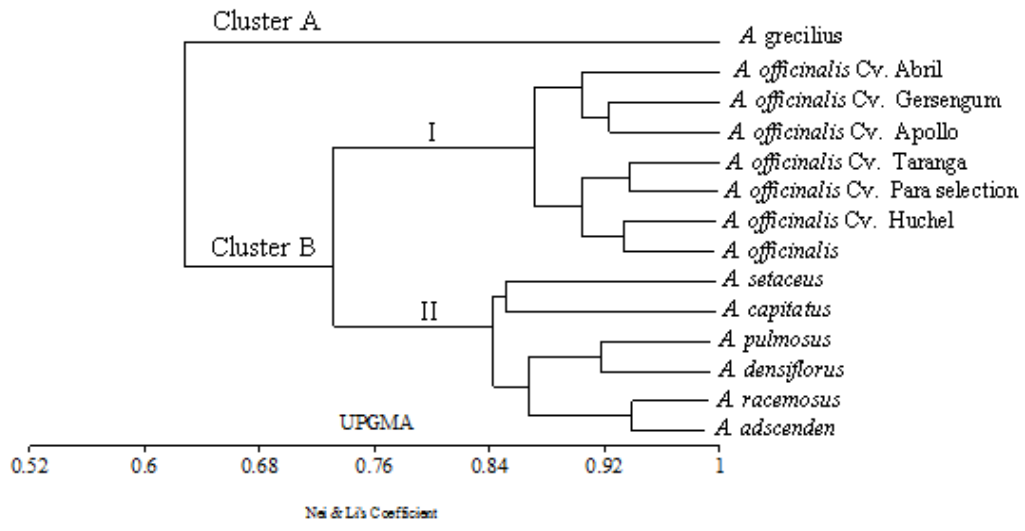

Fig. 5. Cluster analysis of Asparagus species using SSR markers.

Simple sequence repeat information for the transferability of one species to another species was used to determine the genome homology of the species and chance to increase success in PCR amplification utilizing heterologous primer pairs paticularly designed for the plant taxa (Pierantoni et al. 2004). In the present study, fourteen SSR markers were used to determine amplification and polymorphism in other medicinal plants (viz. Saussurea costus and Curcuma longa L.). Of these 14 primers, six primer pairs (42.8\%) showed successful amplification in Curcuma longa L. and Saussurea costus. Our result was consistent with previous studies reported in Jatropha curcas and Catheranthus roseus. In several 
studies, transferability level was found to be only 20-30 \% (Tahan et al. 2009). Thus, SSR markers generated in the present study are satisfactory and provide a new tool for its further use in characterization and genetic variation in the related Asteraceae and Zingiberaceae families (Fig. S2).

Table S2. Genetic similarities index based on Nei and Lie Coefficient using SSR markers for Asparagus species and $A$. officinalis cultivars.

\begin{tabular}{|c|c|c|c|c|c|c|c|c|c|c|c|c|c|}
\hline & 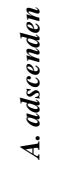 & 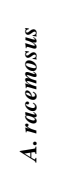 & $\begin{array}{l}\tilde{\Xi} \\
\stackrel{\Xi}{\Xi} \\
\dot{\Xi} \\
\dot{\pi}\end{array}$ & 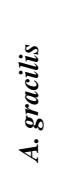 & 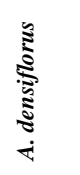 & 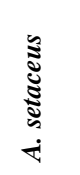 & 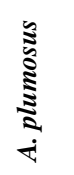 & 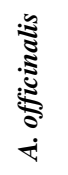 & 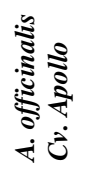 & 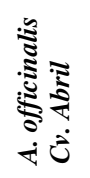 & 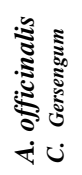 & 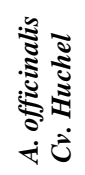 & 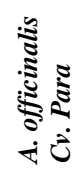 \\
\hline A.racemosus & 0.94 & & & & & & & & & & & & \\
\hline A. capitatus & 0.86 & 0.83 & & & & & & & & & & & \\
\hline A. gracilis & 0.75 & 0.76 & 0.62 & & & & & & & & & & \\
\hline A. densiflorus & 0.89 & 0.87 & 0.78 & 0.75 & & & & & & & & & \\
\hline A. setaceus & 0.83 & 0.85 & 0.85 & 0.70 & 0.84 & & & & & & & & \\
\hline A. plumosus & 0.86 & 0.84 & 0.88 & 0.70 & 0.92 & 0.86 & & & & & & & \\
\hline A. officinalis & 0.75 & 0.73 & 0.84 & 0.59 & 0.68 & 0.78 & 0.74 & & & & & & \\
\hline $\begin{array}{l}\text { A. officinalis } \\
\text { Cv. Apollo }\end{array}$ & 0.73 & 0.71 & 0.82 & 0.56 & 0.69 & 0.80 & 0.76 & 0.93 & & & & & \\
\hline $\begin{array}{l}\text { A. officinalis } \\
\text { Cv. Abril }\end{array}$ & 0.75 & 0.76 & 0.80 & 0.60 & 0.75 & 0.86 & 0.77 & 0.88 & 0.91 & & & & \\
\hline $\begin{array}{l}\text { A. officinalis } \\
\text { Cv. Gersengum }\end{array}$ & 0.72 & 0.74 & 0.82 & 0.54 & 0.72 & 0.79 & 0.78 & 0.86 & 0.92 & 0.90 & & & \\
\hline $\begin{array}{l}\text { A. officinalis } \\
\text { Cv. Huchel }\end{array}$ & 0.70 & 0.72 & 0.76 & 0.57 & 0.71 & 0.77 & 0.73 & 0.93 & 0.89 & 0.87 & 0.85 & & \\
\hline $\begin{array}{l}\text { A. officinalis } \\
\text { Cv. Para }\end{array}$ & 0.63 & 0.64 & 0.71 & 0.52 & 0.63 & 0.73 & 0.69 & 0.89 & 0.88 & 0.86 & 0.88 & 0.92 & \\
\hline $\begin{array}{l}\text { A. officinalis } \\
\text { Cv. Taranga }\end{array}$ & 0.63 & 0.64 & 0.71 & 0.52 & 0.63 & 0.73 & 0.69 & 0.89 & 0.88 & 0.83 & 0.84 & 0.92 & 0.94 \\
\hline
\end{tabular}

The similarity matrix was computed using SSR markers based on Nei and Lie coefficients used in NTSYS software. In the present study, the genetic similarity coefficients ranged from 0.10 to 0.87 revealing a high level of genetic variations among Asparagus species, Curcuma longa and Saussurea costus which was consistent with previous study using RAPD markers, which found genetic similarity matrix ranged from $60.5 \%$ to $88.5 \%$ for a collection of $S$. spontaneum clones (Pan et al. 2004). The genetic similarity highest value was 0.87 between $A$. officinalis and A. capitatus, whereas the genetic similarity lowest value was 0.10 between $A$. adscenden and Curcuma longa. Dendogram based on UPGMA analysis grouped the Asparagus species, Curcuma longa and Saussurea costus into 2 major clusters. Cluster I consists of Asparagus species (A. adsceden, A. capitatus, A. officinalis and A. racemosus). Cluster II consists of Curcuma longa and Saussurea costus. (Table S2 and S3).

Table S3. Genetic similarities index based on Nei and Lie Coefficient using SSR markers.

\begin{tabular}{llllll}
\hline & A. adscenden & A. capitatus & A. officinalis & A. racemosus & Saussurea costus \\
\hline A. adscenden & & & & & \\
A. capitatus & 0.81 & & & & \\
A. officinalis & 0.76 & 0.87 & & & \\
A. racemosus & 0.79 & 0.69 & 0.77 & 0.29 & 0.67 \\
Saussurea costus & 0.19 & 0.22 & 0.28 & 0.17 & \\
Curcuma longa & 0.10 & 0.12 & 0.17 & & \\
\hline
\end{tabular}


To the best of our knowledge, this is the first study in Pakistan to use SSR markers for the determination of genetic diversity among Asparagus species and A. officinalis cultivars. For preserving this valuable plant, more Asparagus samples should be gathered, cultivated and domesticated in collections. Prohen et al. (2008) showed that Asia, Africa and Europe are the main centers for the genetic diversity of edible Asparagus. The results of current study showed that Pakistan could be considered as another center for the genetic diversity of Asparagus.

The present study showed that SSR markers developed in Asparagus by in-silico methods is more effective and laborious as compared to classical methods. These in-silico methods play a major role in contributing to the evolution and progress of SSR development. The present study revealed that SSR markers are sufficiently useful and powerful to assess genetic relationships and diversity analysis in Asparagus and A. officinalis cultivars. SSR method effectively discriminates Asparagus species and A. officinalis cultivars. These SSR markers will be particularly useful for evolutionary and genetic mapping studies of Asparagaceae, Asteraceae and Zingiberaceae.

\section{ACKNOWLEDGEMENTS}

We are grateful to Dr. Maryam Shinwari (Quaid-E-Azam University) and Mr. Maazullah (Agriculture Research Institute) for the facilities provided during sampling of Asparagus species and cultivars. The authors acknowledge the technical support of all technicians at the Department of Biotechnology and Genetic Engineering, KUST, Kohat, Pakistan.

\section{REFERENCES}

Altschul, S. F., T. L. Madden, A. A. Schaffer, J. H. Zhang, Z. H. Zhang, W. Miller and D. J. Lipman. 1997. Gapped BLAST and PSIBLAST: a new generation of protein database search programs. Nucleic Acids Res. 25: 3389-3402.

Beckmann, J. S. and J. L. Weber. 1992. Survey of human and rat microsatellites. Genomics. 12: 627-631.

Bornet, B. and M. Branchard. 2001. Nonanchored inter simple sequence repeat (ISSR) markers: Reproducible and specific tools for genome fingerprinting. Plant Mol. Biol. Rep. 19(3): 209-215.

Caruso, M., T. F. Claire, L. Mikeal and Roose. 2008. EST-SSR markers for Asparagus genetic diversity evaluation and cultivar identification. Mol. Breed. 21: 195-204.

Dow, B. D., M. V. Ashley and H. F. Howe. 1995. Characterization of highly variable (GA/CT) n microsatellites in the bur oak (Quercus macrocarpa). Theor. Appl. Genet. 91: 137-141.

Doyle, J. J. and J. L. Doyle. 1987. A rapid DNA isolation procedure for small quantities of fresh leaf tissue. Focus. 12: 13-15.

Dutta, I. C. 2007. Non timber forest products of Nepal (identification, classification, ethnic uses and cultivation). Institute of Forestry, Pokhara, Nepal. 44: 436-438.

Eisen, J. A. 1999. Mechanistic basis for microsatellite instability. In: D. B. Goldstein and C. Schlotterer (eds). Microsatellites: Evolution and applications. Oxford University Press, Oxford, UK., pp. 34-48.

Ginwal, H. S., M. Neha, S. M. Shalini, B. Santan and B. Preeti. 2011. Genomic DNA isolation and identification of Chloroplast microsatellite markers in Asparagus racemosus Wild. Through cross amplification. Ind. J. biotechnol. 10: 33-38.

Gupta, S. and M. Prasad. 2009. Development and characterization of genic SSR markers in Medicago truncatula and their transferability in leguminous and non-leguminous species. Genome. 52: 761-771.

Hafezi-Shahroodian, S., D. Azadfar, H. Soltanloo and S. Ramezanpour. 2011. Genetic variability in natural Iranian populations of Cupressus sempervirens var. horizontalis in Caspian Sea coastward assessed by SSR markers. Plant Omics. J. 4(1): 19-24. 
Huang, X. and A. Madan 1999. CAP3: a DNA sequence assembly program. Genome Res. 9: 868-877.

Koppolu, R., D. U. Hari, L. D. Sangam, A. H. David and K. V. Rajeev. 2010. Genetic relationships among seven sections of genus Arachis studied by using SSR markers. BMC Plant Biol. 10: 15.

Kubitzki, K. and P. J. Rudall. 1998. Asparagaceae. In: K. Kubitzki (ed.). The families and genera of vascular plants. Monocotyledons: Lilianae (except Orchidaceae). Vol. 3. Springer-Verlag, Berlin, Germany., pp. 125126.

Kumpatla, S. P. and S. Mukhopadhyay. 2011. Mining and survey of simple sequence repeats in expressed sequence tags of dicotyledonous species. Genome. 48: 985-998.

Mandal, S. C., C. K. A. Kumar, M. S. Lakshmi, S. Sinha, T. Murugesan, B. P. Saha and M. Pal. 2007. Antitussive effect of Asparagus racemosus root against sulfur dioxide induced cough in mice. Fitoterapia. 71: 686-689.

Nei, M. and W. Li. 1979. Mathematical model for studying genetic variation in terms of restriction endonucleasis. Proc. Natl. Acad. Sci. 76: 5269-5273.

Pan, L., X. Qiuju, Q. Zhiwu, L. Honggao, K. Weidong and D. Yi. 2010. Development of Novel EST-SSRs from Sacred Lotus (Nelumbo nucifera Gaertn) and Their Utilization for the Genetic Diversity Analysis of $N$. nucifera. J. Hered. 101(1): 71-82.

Perez, F., O. Juan, Z. Mariuxi, G. Cesar, C. Jorge and F. A. M. J. Volckaert. 2005. Development of EST-SSR Markers by Data Mining in Three Species of Shrimp: Litopenaeus vannamei, Litopenaeus stylirostris, and Trachypenaeus birdy. Marin Biotechnol. 7: 554-569.

Pierantoni, L., K. H. Cho, I. S. Shin, R. Chiodini, S. Tartarini, L. Dondini, S. J. Kang and S. Sansavini. 2004. Characterization and transferability of apple SSRs to two European pear F1 populations. Theor. Appl. Genet. 109: 1519-1524.

Portis, E., I. Nagy, Z. Sasva, A. Stagelri, L. Barchi and S. Lanteri. 2007. The design of Capsicum spp. SSR assays via analysis of In silico DNA sequence, and their potential utility for genetic mapping. Plant Sci. 172: 640648.

Prohen, J., F. Nuez and M. J. Carena. 2008. Handbook of plant breeding. Springer Publlishing. 364p.

Ray, S., J. M. Madhumita and M. Sandip. 2010. Phylogenetic Relationship among six Economically Important Species of Asparagus Utilizing RAPD, ISSR and Isozyme Polymorphism. Biores. Bull. 3: 153-160.

Rozen, S. and H. J. Skaletsky. 2000. Primer3 on the WWW for general users and for biologist programmers. In: S. Krawetz and S. Misener (eds.). Bioinformatics Methods and Protocols: Methods in Molecular Biology. Humana Press, Totowa, NJ., pp. 365-386.

Shasnay, A. K., M. P. Darokar, D. Sakia, S. Rajkumar, V. Sundaresan and S. P. S. Khanuja. 2003. Genetic diversity and species relationship in Asparagus spp. using RAPD analysis. J. Med. Arom. Plant Sci. 25: 698704.

Tahan, O., Y. P. Geng and L. Y. Zeng. 2009. Assessment of genetic diversity and population structure of Chinese wild almond, Amygdalus nana, using EST- and genomic SSRs. Biochem. Syst. Ecol. 37: 146-153.

Thiel, T., W. Michalek, R. K. Varshney and A. Graner 2003. Exploiting EST databases for the development and characterization of gene-derived SSR-markers in barley (Hordeum vulgare L.). Theor. Appl. Genet. 106: 411-422.

Varshney, R. K., T. Thiel, N. Stein, P. Langridge and A. Graner. 2002. In silico analysis on frequency and distribution of microsatellites in ESTs of some cereal species. Cell Molecul. Biol. 7: 537-546.

Victoria, F. C., C. Luciano and C. O. Antonio. 2011. In silico comparative analysis of SSR markers in plants. BMC Plant Biol. 11: 15.

Wen, M., W. Haiyan, X. Zhiqiang, Z. Meiling, L. Cheng and W. Wenquan. 2010. Development of EST-SSR and genomic-SSR markers to assess genetic diversity in Jatropha Curcas L. BMC Res Note. 3: 42. 